\title{
Phase Transition in Liquid Drop Fragmentation
}

\author{
Cristian F. Moukarzel * Silvia F. Fernández-Sabido ${ }^{\dagger}$ and J. C. Ruiz-Suárez ${ }^{\ddagger}$ \\ CINVESTAV del IPN Unidad Mérida, \\ Depto. de Física Aplicada, \\ 97310 Mérida, Yucatán, México
}

(Dated: September 20, 2018)

\begin{abstract}
A liquid droplet is fragmented by a sudden pressurized-gas blow, and the resulting droplets, adhered to the window of a flatbed scanner, are counted and sized by computerized means. The use of a scanner plus image recognition software enables us to automatically count and size up to tens of thousands of tiny droplets with a smallest detectable volume of approximately $0.02 \mathrm{nl}$. Upon varying the gas pressure, a critical value is found where the size-distribution becomes a pure power-law, a fact that is indicative of a phase transition. Away from this transition, the resulting size distributions are well described by Fisher's model at coexistence. It is found that the sign of the surface correction term changes sign, and the apparent power-law exponent $\tau$ has a steep minimum, at criticality, as previously reported in Nuclear Multifragmentation studies [1, 2]. We argue that the observed transition is not percolative, and introduce the concept of dominance in order to characterize it. The dominance probability is found to go to zero sharply at the transition. Simple arguments suggest that the correlation length exponent is $\nu=1 / 2$. The sizes of the largest and average fragments, on the other hand, do not go to zero but behave in a way that appears to be consistent with recent predictions of Ashurst and Holian [3, 4].
\end{abstract}

PACS numbers: 64.60.-i, 64.60.Ak, 46.30.Nz

\section{INTRODUCTION}

Fragmentation processes are important in several different fields, including powder technology [5, 6], soil physics 7, 8, 9, 10] nuclear physics [11, 12], astrophysics [13, 14], genetics [15, 16], sprays 17, 18, 19], fuel combustion 20, 21], brittle fracture [22, 23, 24] and geology [25, 26], just to name a few.

Because of the nature of the fragmentation process, only the final distribution of fragment sizes is usually observable, thus becoming the main quantity of interest. Size-distributions have been characterized by different empirical and theoretical statistical laws in the past. The lognormal distribution can be expected whenever a breakdown process can be described as a multiplicative cascade, and has been applied in many fragmentation examples 21, 24, 27, 28, 29]. Simmons' law 30, 31, 32 appears to hold universally for sprays, and states that the mass distribution is Gaussian when written in terms of the square root of drop diameters. Villermaux [17, 18, 33] discusses the breakup process of ligaments in liquid jets, and concludes that each ligament gives rise to a distribution of droplet sizes that is essentially a Gamma distribution. The Weibull distribution can be derived from first-principles for the fragmentation of solids by cracking [34, 35], and reads $\% N(>m)=e^{-(m / \hat{m})^{q}}$, where $\% N(>m)$ is the fraction of droplets with mass larger than $m$. Brown and

\footnotetext{
*Corresponding author: cristian@mda.cinvestav.mx

${ }^{\dagger}$ Present address: Laboratoire Informatique d'Avignon, France. ${ }^{\ddagger}$ Present address: Cinvestav-Monterrey, Av. Cerro de las Mitras 2565, Monterrey NL, 64060, México
}

Wohletz [34] remark that the Weibull distribution may look similar to the lognormal, and suggest that the apparent goodness of fits using lognormal could be fortuitous in some cases. Other authors have used the (empirical) Rosin-Rammler form [6, 34, 36], which applies to the accumulated mass density in clusters of diameter larger than $D$, and states that $\% M(>D)=e^{-\left(D / D_{m}\right)^{q}}$.

In contrast with these approaches, which postulate exponential, or else rapidly decaying size distributions, Oddershede et al 37] have claimed that the size-distribution of fractured objects is an inverse power-law, and furthermore suggested that a form of self-organized criticality (SOC) [38] might be responsible for this. Powerlaw distributions are often found right at second order phase transitions, in which case one or more parameters must take precisely defined values. However there are systems whose dynamics converges to a stationary state that is critical, a phenomenon termed SOC [38] and for which no parameter tuning is needed. Later investigations 39, 40, 41, 42, 43, 44] report "composite" powerlaws, or transitions between lognormal and power-laws as the fragmentation energy is increased [21, 229, 45].

Recent works 22, 46, 47, 48, 49] have reported that fragmentation processes are only critical (and the ensuing size-distribution a power-law) at a precisely defined value of the impact energy. Within this picture, the fragmentation phenomenon goes through a nonequilibrium phase transition at some well-defined value of the impact energy. Evidences of criticality at a well defined energy where also found in nuclear multifragmentation (NMF) [11, 12] experiments, and have been interpreted in the context of a percolative transition 50 , or else in relation with liquid-gas coexistence [2, 51, 52, 53, 54, 55, 56, 57, 58] in nuclear matter. In this work, water-glycerine drops are broken by a 
controlled-pressure gas blow, and the resulting droplets are counted and sized by computerized methods that involve scanning and image-processing. The only control parameter, the gas pressure, varies between 11 and 100 psi, and this in turn determines the amount of energy available for the breakdown event. Various statistical measures characterizing the resulting size-distributions are obtained and analyzed, for a minimum of ten and a maximum of fifty breakdown events at each pressure. Our main finding is the existence of a phase transition around $P_{c} \approx 17 \mathrm{psi}$, where the size-distribution becomes a pure power-law with an exponent $\tau_{\text {min }} \approx 1$ within a limited size-range. Away from $P_{c}$, the size distribution is well described by the product of a power-law and an exponentially decaying term (Eq. (4)), as given by Fisher's model at coexistence [59].

The rest of this article is organized as follows. In Section III a brief discussion is done of some theoretical approaches which have been used to characterize sizedistributions in the past. Section III describes our experimental apparatus, as well as the data processing techniques we use to obtain statistics on size distributions. Our results are presented in Section IV. In Section IVA the resulting size distributions are characterized by means of a simplified Fisher expression (Eq. (4)), essentially a power law with an exponential cutoff. The total surface is discussed in Section IVB. Section IVC discusses whether the observed transition can be characterized as percolative. The mass of the largest fragment (Section IVC1) and moment correlations (Section IVC2 do not support such interpretation. Section IVD discusses the behavior of the largest and average mass fragment in the context of recent theoretical elaborations [3, 4]. In Section IVE the concept of dominance is introduced and applied to our data. Finally, our results are discussed in Section V.

\section{SIZE DISTRIBUTIONS}

In recent years, several brittle fragmentation [22, 23, 49] and NMF [11, 50] experiments have been interpreted in the context of Percolation Theory [60]. A percolation transition is claimed to exist between a "connected" phase and "disconnected" phase, as the impact energy is increased. In the connected phase, a large cluster (drop, fragment) exists after the fragmentation event, that contains a significant fraction of the total mass. In the disconnected phase no such large cluster exists. The natural order parameter for a percolative transition of this type is the average size of the largest fragment. Measurements of this order parameter [23, 50] have shown that it goes to nearly zero at and above the transition, in a way that is consistent with the proposed interpretation.

At the percolation critical point, the number distribution $N(x)$ of clusters of mass $x$ becomes a power-law $N(x) \sim x^{-\tau}$, where the exponent $\tau$ takes values close to 2.3 in three dimensions. NMF experiments have been reported to give results consistent with $\tau \approx 2.3[11,50]$ as well, while brittle fragmentation experiments seem to produce different values of $\tau$.

NMF experiments are sometimes more generally discussed [53, 61, 62, 63, 64, 65, 66] in the context of Fisher's Droplet Model [59], which states that the number of clusters (droplets, nuclei) of mass $x$ is given by

$$
N(x)=N_{0} x^{-\tau} e^{B(p, T) x-C(p, T) x^{\sigma}},
$$

where $\tau$ and $\sigma$ are independent critical exponents. Coefficients $B(p, T)$ and $C(p, T)$ depend on surface tension, temperature, and chemical potentials in the "liquid" and "gas" phases:

$$
B(p, T)=\frac{\Delta \mu}{T}=\frac{\mu_{g a s}-\mu_{l i q}}{T},
$$

and

$$
C(p, T)=\frac{c_{0} \epsilon}{T}
$$

Here $c_{0}$ is a constant that depends on surface tension, and $\epsilon=\left(T_{c}-T\right) / T_{c}$ measures the departure from the critical temperature.

The term $B(p, T) x$ measures the bulk contribution to the free energy of a droplet of size $x$. In a $(p, T)$ phase diagram, $B<0$ if the "Gas" phase is thermodynamically stable, i.e. its chemical potential is lower than that of the "cluster", or "liquid" phase.

The term $C x^{\sigma}$ measures the surface energy of a droplet of size $x$, so that $\sigma$ is the critical exponent that relates the dimension of the surface to that of the volume of a droplet. For compact droplets in 3 d one has $\sigma=2 / 3$. For the liquid-gas transition $\sigma$ takes values close to $2 / 3$. On the coexistence line, $\mu_{l}=\mu_{g}$ and thus $B=0$, so

$$
N(x)=N_{0} x^{-\tau} e^{-C x^{\sigma}} .
$$

Furthermore, at the critical point one has $C=0$, and the size-distribution becomes a pure power-law with exponent $\tau$.

\section{EXPERIMENTAL SETUP AND DATA ACQUISITION}

\section{A. Setup}

Our experimental setup is schematically shown in Fig. 1. An open, straight glass tube (T) of $6 \mathrm{~cm}$ length and $7 \mathrm{~mm}$ inner diameter, has a solid coaxial glass rod (1mm diameter) attached to its inner surface, with the rod's tip protruding $5 \mathrm{~mm}$ downwards from the lower end of the tube. From this tip hangs a small (11.5 $\mu l[71])$ liquid drop (D) of an opaque solution obtained by mixing equal quantities of glycerol and water, plus a small amount of water-soluble black dye. The upper end of this glass tube is connected to a pressurized chamber $(\mathrm{C})$ 
via a $40 \mathrm{~cm}$ length plastic (H1) hose, and isolated from it by a solenoid valve (SV). The tube is fixed so that the droplet hangs $15 \mathrm{~cm}$ above the glass window of a flatbed scanner.

A nitrogen cylinder is connected to the chamber through

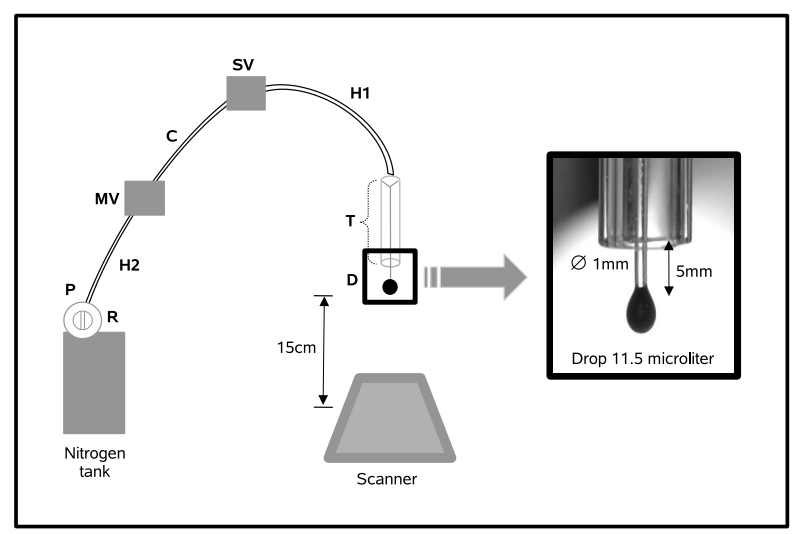

FIG. 1: Experimental Setup

a plastic hose (H2), and a pressure regulator $(\mathrm{R})$, which is provided with a manometer $(\mathrm{P})$. The chamber is formed by a $10 \mathrm{~cm}$ length, $3 / 8$ inch inner diameter plastic hose and is limited by two bypass valves, one manual (MV), the other electromagnetic (SV).

For each droplet breakdown experiment, the nitrogen pressure was selected with regulator $\mathrm{R}$, and the manual valve was opened until the pressure in chamber $\mathrm{C}$ equilibrated, after which it was closed again. Then, the electromagnetic valve was opened by means of an electric switch, releasing the gas contained in chamber $\mathrm{C}$, which then flowed through tube $\mathrm{T}$, fragmenting the droplet and projecting its fragments downwards. The resulting droplets, adhered to the scanner's glass window, were scanned and subsequently counted and sized with the help of specialized computer software.

Before each breakdown experiment, the scanner glass was treated with a hydrophobic solution containing Teflon. Because of this treatment of the glass surface, the contact angle of the liquid onto the glass was close to 90 degrees, so that the droplets turned out to be very similar in shape to semi-spheres. After the fragmentation, a thin spacing was inserted between the scanner lid and its glass window, and a high-resolution (600 dpi) scan was performed. Several (from 10 to 50) fragmentation experiments were carried out varying the jet pressure $\mathrm{P}$, from 11 psi to 100 psi. The number of droplets obtained varied, from a few ones at 11 psi to tens of thousands at 100 psi.

\section{B. Data Processing}

After each fragmentation, a scanning operation was performed. All scanned images were obtained at 600 dpigrayscale, and stored using a lossy compressed graphic format, for later processing. Because of the nonnegligible height of the droplets, most images contained faint shadows, which were removed by image thresholding [72]. Subsequent image processing and droplet identification, counting, and sizing, was performed with the help of ImageJ 73 ]

The output from ImageJ consisted essentially of a set

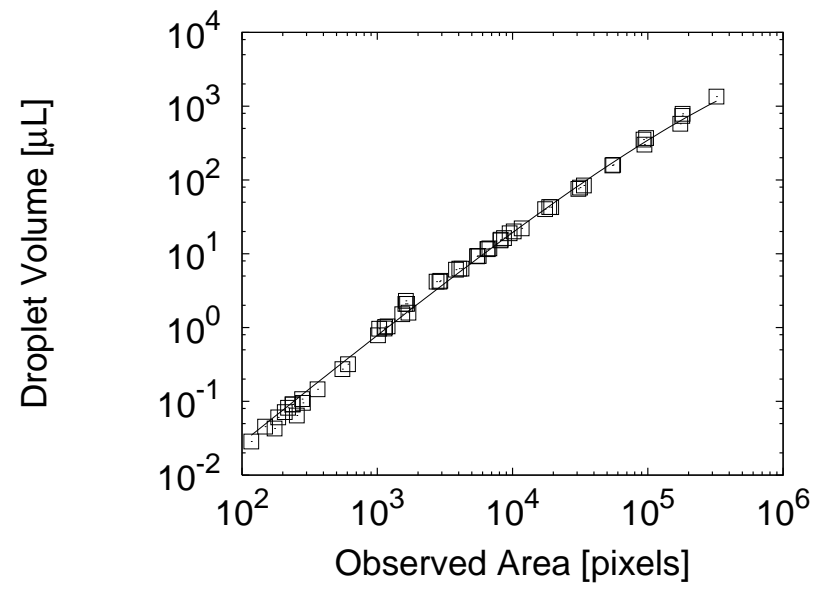

FIG. 2: Data used for fitting Eq. (6) (squares) and the resulting fit (solid line).

of droplet areas, in pixels. In order to estimate droplet volumes $V_{i}$ from the observed areas $\Omega_{i}$, we did the following. If gravity effects were negligible then all droplets would be plane sections of a sphere and their volumes $V_{i}$ would be given by

$$
V_{i}=\lambda_{1} \Omega_{i}^{3 / 2},
$$

with $\lambda_{1}$ some constant. However (5) is a good approximation only for the tiniest droplets, whose shapes are less affected by gravity. Larger droplets suffer a significant flattening and therefore Eq. (5) must be corrected. Under the effect of gravity, the height $h(\Omega)$ of a liquid droplet on a flat horizontal surface is proportional to $\sqrt{\Omega}$ when $\Omega$ is small, but saturates to a constant value $h_{\infty}$ for large values of $\Omega$, in which case the fluid forms a flat "lake" of approximately constant height. Thus $V$ satisfies (5) for small $\Omega$, but is proportional to $\Omega$ for large $\Omega$. Assuming, for simplicity, a stretched exponential crossover, we write

$$
V(\Omega)=\lambda_{1} \Omega^{3 / 2} e^{-\left(\Omega / \Omega_{0}\right)^{\beta}}+\lambda_{2} \Omega\left[1-e^{-\left(\Omega / \Omega_{0}\right)^{\beta}}\right],
$$

where $\lambda_{1}, \lambda_{2}, \Omega_{0}$, and $\beta$ are to be obtained from fits of calibration data (See Fig. (2). The resulting values where $\lambda_{1}=3.15 \times 10^{-5}, \lambda_{2}=8.3 \times 10^{-7} \Omega_{0}=10^{5}$ pixels, and $\beta=0.366$. In order to obtain the data required for the fit, the projected areas were measured for several droplets having known volumes. Their volumes were obtained using calibrated pipettes (for the larger ones), and glass capillaries (for the smaller ones). The data used for the fit, together with the resulting fit, are displayed in Fig. 2. 
Our final expression for the volume/area relationship extrapolates to $V \sim 2 \times 10^{-5} \mu \mathrm{l}$ when $\Omega=1$ pixel, implying that the smallest detectable volume is $V_{\min }=0.02 \mathrm{nl}$. Eq. (6), together with the parameter values obtained

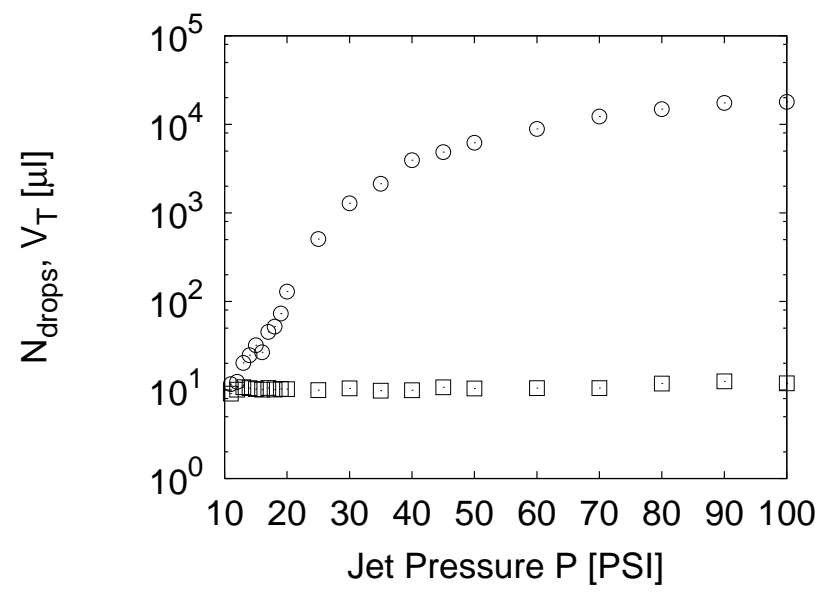

FIG. 3: Average total observed volume $V_{T}$ (squares) and average number of droplets $N_{\text {drops }}$ (circles) as a function of jet pressure $P$. The initial volume of the droplet is $11.5 \mu \mathrm{l}$.

from the fit, were embedded into a $\mathrm{C}$ program, which read droplet areas (as calculated by ImageJ), and used this expression to calculate volumes from them, additionally implementing the statistical analysis of size distributions to be described in Section IV.

\section{RESULTS}

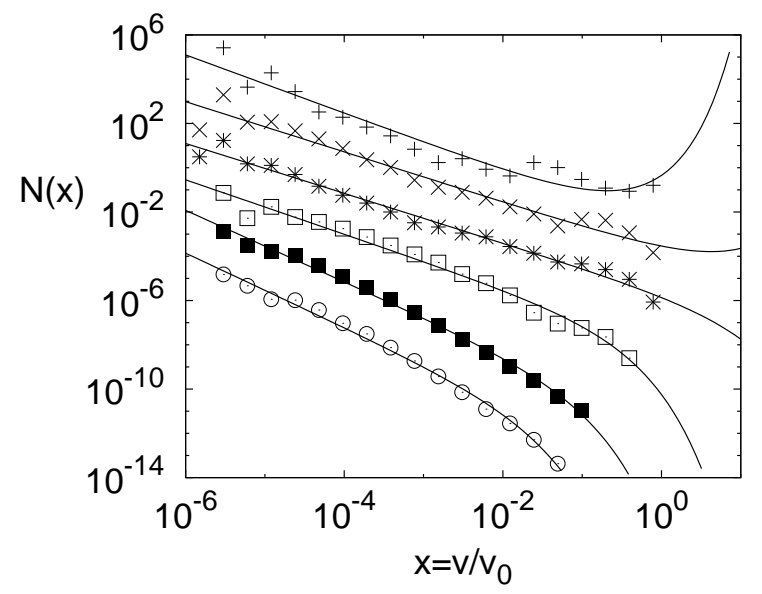

FIG. 4: $N(x)$, the number of drops of relative mass $x=V / V_{0}$, for (from top to bottom) $P=11,15,17,25,60$, and 100 PSI. Solid lines are fits of Eq. (4) with $\sigma=2 / 3$. Plots have been displaced downwards for clarity.

For each breakdown event, a set of droplet volumes $V_{i}$, $i=1,2, \ldots, n$ was obtained as described in Section IIIB. and the moments $M_{k}=\sum_{i} x_{i}^{k}$ were calculated in terms of $x_{i}=V_{i} / \sum_{j=1}^{n} V_{j}$, the droplet volume fractions. The average number of droplets, as well as the average total volume $V_{T}=\sum_{i} V_{i}$, are displayed in Fig. 3 as a function of jet pressure $\mathrm{P}$. The fact that $V_{T}$ remains almost constant and equal to the initial volume confirms that most of the mass resulting from each fragmentation gets caught by the scanner window, i.e. that there are no significant mass losses, even at the largest pressures considered.

\section{A. Size distributions}

Fig. 4 shows log-log plots of the normalized fraction of droplets $N(x)$ with volume fraction $x$, for several values of the breakup pressure $P$. Continuous lines in Fig. 4 are the result of fitting Fisher's Model in the form indicated by Eq. (4), which is appropriate for the coexistence region. We fixed $\sigma=2 / 3$ and adjusted $N_{0}(P)$ (not shown), $C(P)$ (Fig. 5) and $\tau(P)$ (the apparent exponent, see Fig. 6) independently for each pressure $P$. We checked that the qualitative features of these fits did not change much for $\sigma$ ranging from $1 / 3$ to 2 .

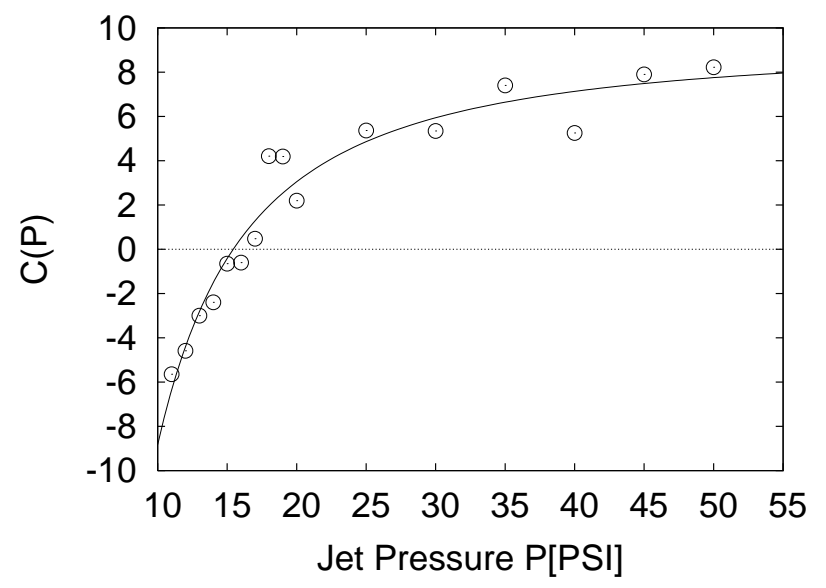

FIG. 5: Amplitude $C(P)$ (circles) of surface correction term in Eq. (4), for $\sigma=2 / 3$. The solid line is an empirical fit (see text).

The "surface" correction term $C(P)$ (Fig. 50) changes sign around $P_{0}=17$ PSI, implying that the size distribution is similar to a pure power-law there, and giving the first hint for the existence of a phase transition. The resulting curvature change that is apparent in Fig. 4 has been reported and discussed in NMF experiments as well [53, 56]. The solid line in Fig. 5 is a fit of the form

$$
C(P)=a\left[\left(\frac{P}{P_{0}}\right)^{\mu}-1\right]
$$

and results in $P_{0}=15.5$ and $\mu=-1.5$. This suggests that

$$
C \sim\left(P-P_{0}\right)
$$




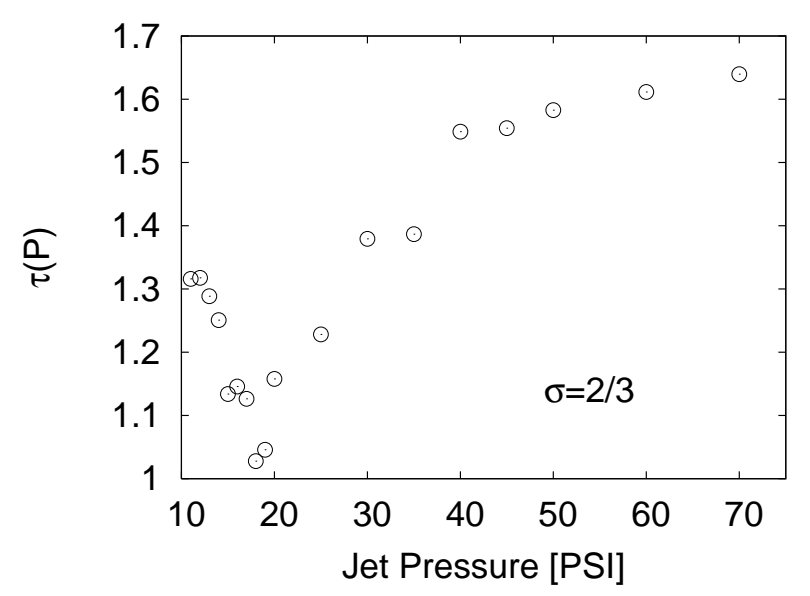

FIG. 6: Fits of Fisher model (Eq. (4)) to the droplet density $N(x)$ (Fig. 4), produce an apparent exponent $\tau$ (circles) showing a deep minimum $\tau_{\text {min }} \approx 1$ around 18 PSI.

near the crossing point $P_{0}$.

The exponential term in (44) can be written, as is usually done in the scaling description of critical phenomena, as

$$
e^{-C x^{\sigma}}=F_{ \pm}\left(\left(x / x_{0}\right)\right)
$$

where the subscript \pm means $P>P_{0}$ or $P<P_{0}$ respectively. The "cutoff" $x_{0}$ satisfies

$$
x_{0}=|C|^{-1 / \sigma} .
$$

For compact droplets we can furthermore write

$$
x_{0}=\xi^{3}
$$

in three dimensions, where $\xi$ is a correlation length (the linear size of the typical droplet). Considering (8), (10), and (11) one finds that, near $P_{0}$,

$$
\xi \sim\left|P-P_{0}\right|^{-\nu}
$$

with $\nu=1 / 2$, i.e. a classical value for a thermal critical exponent. Of course, this specific result $(\nu=1 / 2)$ depends on the validity of (8), which in view of the quality of our data cannot be taken for granted.

The apparent exponent $\tau(P)$ (Fig. 6) goes through a steep minimum $\tau_{\text {min }} \approx 1$ around 18 PSI. A similar minimum in $\tau$ has been taken as an indicator for the location of a phase transition in NMF experiments [1, 2] in the past. In fact, Richert and Wagner [11] define the critical temperature $T_{c}$ as the one that minimizes $\tau(T)$. We may thus consider this steep minimum as a further indication of a phase transition in the breakdown process, occurring somewhere in the range 16-18 PSI for our specific experimental setup.

\section{B. Excess surface}

The total normalized surface $\Omega_{F}=\sum_{i} x_{i}^{2 / 3}$ of the final set of drops in each breakdown process gives an estimation of the total amount of energy transferred to the system of drops (i.e. neglecting kinetic energy) and is thus an interesting physical quantity in the breakdown experiment. We define the relative excess surface as

$$
E(P)=\frac{\Omega_{F}-\Omega_{0}}{\Omega_{0}}
$$

where $\Omega_{0}=1$ is the initial surface. By taking averages over breakdown events at pressure $P$, the results displayed in Fig. 7 are obtained. It can be seen in this figure that the pressure-dependence is approximately linear everywhere, but there is a sharp discontinuity in its slope, again happening around 17 PSI. Fitting two straight lines intersecting at $P_{0}$ to the data shown in Fig. 7 (dotted line) one obtains the estimate $P_{0}=17.6$ PSI.

If the excess surface is interpreted as an "energy" and the jet pressure as a measure of the effective "temperature", the discontinuous slope in Fig. 7 is analogous to a discontinuity in the specific heat.

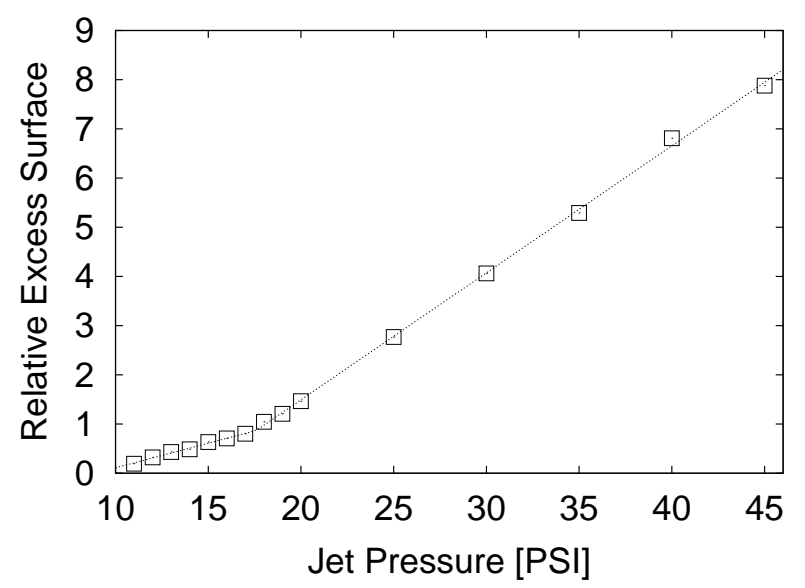

FIG. 7: Surface increase versus Pressure has a discontinuous derivative around 17.6 PSI. The dotted line is a linear fit, with the location of the kink as a fitting parameter.

\section{Is the observed transition percolative?}

\section{Largest fragment}

Recently there have been suggestions that brittle fragmentation [22, 23, 49] and NMF [11, 50] experiments can be rationalized in the context of a percolative [60] phase transition. The validity of such interpretations rests, to a great extent, upon the identification of a relevant order parameter which has the properties of the infinitecluster density in percolation. Within this picture, the connected or percolating phase must be characterized by the existence of macroscopic fragments, while no such fragments must exist in the disconnected phase (at large breakdown energies). The average size of the largest fragment, which goes to zero abruptly at the critical energy, 
plays the role of an order parameter in brittle fragmentation [22, 23, 49], so it is natural to first consider the largest droplet size as a tentative order parameter also in the case of our experiments.

The average mass $Z_{\max }$ of the largest fragment is shown

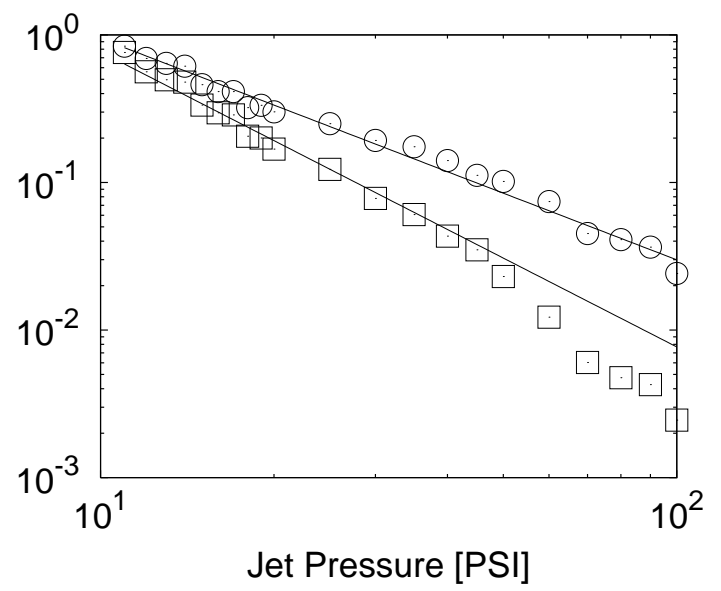

FIG. 8: Maximum droplet size $Z_{\max }$ (circles) and average droplet size $Z_{a v g}=M_{2}$ (squares), as a function of jet pressure $P$. Straight lines are of the form $c_{1} P^{-2}$ and $c_{2} P^{-3 / 2}$ respectively. Assuming that the system's expansion speed $\eta$ is proportional to the jet pressure $P$, the observed dependencies $Z_{\text {max }} \sim P^{-3 / 2}$, and $Z_{\text {avg }}=M_{2} \sim P^{-2}$ are consistent with recent predictions of a simple energy-balance theory of Ashurst and Holian [3, 4, 47].

in Fig. 8 from our droplet fragmentation experiments. It turns out that $Z_{\max }(P)$ has a smooth pressure dependence of the type $Z_{\max }(P) \sim P^{-3 / 2}$, without any traces of a singular behavior. Notice that, if there where a percolative transition, the mass of the largest droplet (the infinite cluster) should go to zero at the critical point. Therefore we conclude that the behavior of $Z_{\max }$ does not support the existence of a percolative transition in this system.

\section{Moment correlations}

In his discussion of NMF experiments, Campi [50] notices that correlations among some moments of the size distribution are similar to those found in Percolation. In particular, a plot is presented of $Z_{\max }$ versus $S_{2}=M_{2} / M_{1}$ (moments are calculated excluding the contribution of the largest cluster) and it is argued that the rounded "nose" of events with large values of $S_{2}$ corresponds to the percolative "critical region".

Fig. 9 shows a scatter plot of $Z_{\max }$, the largest cluster's mass, versus $S_{2}$, for each fragmentation event in our experiments. This plot is similar in many aspects to the one considered by Campi [50], in particular with respect to the existence of a "nose" of events with large values of $S_{2}$. It can be easily seen, however, that this feature by itself is not indicative of critical behavior. In order to see

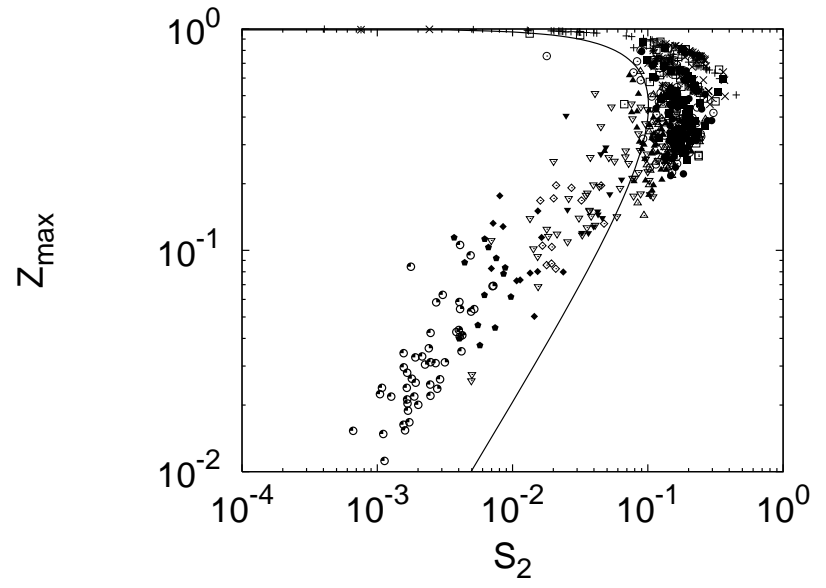

FIG. 9: Inclusive (all pressures) scatter plot of the mass $Z_{\max }$ of the largest piece versus $S_{2}=M_{2} / M_{1}$, where the moments have been calculated without the contribution of the largest piece. Points near the "nose" of events with large values of $S_{2}$ correspond to pressures in the range 16-18 PSI. Notice, however, that the existence of this feature is by itself not indicative of critical behavior, since it is there even for the trival partition described in the text (solid line).

this, consider the breakup of a droplet of unitary volume into an infinite succession of droplets $i=0,1,2, \ldots$ of volume $v_{i}=(1-\lambda) \lambda^{i}$, for $0<\lambda<1$. As $\lambda$ goes from almost zero to almost one, the resulting size distribution changes from being dominated by essentially one large drop, to being composed of a "mist" of tiny droplets. Clearly there is no phase transition in this system.

For this simple partition, the largest mass is $Z=v_{0}=$ $(1-\lambda)$. Furthermore, it is straightforward to show that $S_{2}=Z(1-Z) /(2+Z)$. A plot of $S_{2}\left(Z_{\max }\right)$ for this simple partition is displayed in Fig. 9 (thick line). It can be seen that its overall features are the same as reported by Campi [50], i.e. $S_{2}$ goes through a maximum and then decreases, as $Z_{\max }$ decreases $(\lambda$ increases). Thus we conclude that the rounded "nose" in these plots is not by itself indicative of a phase transition, and cannot be taken as evidence of percolative behavior.

\section{Ashurst and Holian theory}

Recently Ashurst and Holian (AH) [3, 4] (see also Ästrom, Holian and Timonen [47]), considering a $d$ dimensional fluid system that expands uniformly at rate $\eta$, predicted that the maximum droplet size $Z_{\max }$ should behave as $\eta^{-d / 2}$ while the average droplet size $Z_{\text {avg }}$ should decrease as $\eta^{-2 d / 3}$ with increasing expansion rate 74 .

Since in our experiments the expansion rate $\eta$ is not a control parameter, the applicability of AH results is not obvious. However we find (See Fig. 8) that $Z_{\max } \sim P^{-1.5}$ and $Z_{\text {avg }} \sim P^{-2}$, which is consistent with AH predictions in 3 dimensions, if the (unknown) expansion rate $\eta$ is as- 
sumed to be proportional to the jet pressure $P$.

\section{E. Dominance Probability}

We now set to try and identify the nature of the phase transition at 17 PSI, by defining a useful order parameter that we call dominance probability. Although, as seen already, the largest fragment size is a smooth function of $P$, we have noticed that, at low pressures, most of the mass is concentrated in a few large fragments, while at larger pressures the mass is evenly distributed among a large number of small droplets. More precisely, at very low pressures the mass distribution appears to be composed of one large fragment plus many tiny droplets. Upon increasing the jet pressure $P$, often two large droplets appear, next events with three large fragments become prevalent, and so on. At large enough pressures, however, the original droplet mass is more evenly distributed among a large number of droplets, without a clear size distinction between large and small ones. We then seek to define an order parameter that quantifies the property that the mass distribution be "dominated" by a few large fragments or not.

For an arbitrarily defined "dominance factor" $\gamma$ taking values slightly smaller than 1 (one can take e.g. $\gamma=0.9$ to exemplify the ideas), we say that there is dominance at level 1 if the largest fragment's mass is larger than $\gamma$ times the total mass. If this condition is not satisfied, the largest fragment is removed and we check whether the second largest fragment's mass is larger than $\gamma$ times the total remaining mass (after removing the first largest). If this is the case, we say that there is dominance at level 2. If not, the second largest droplet is removed and we proceed to do the same check with the third largest one, and so forth, until only the last droplet remains.

These ideas can be formalized as follows. First all droplets in a given fragmentation event are ordered by size: $x_{1}>x_{2}>\ldots>x_{n}$. Then for a given value of the "dominance parameter" $\gamma$, check whether the dominance condition

$$
x_{k}>\gamma \sum_{k}^{n} x_{i}
$$

is satisfied for some $k<n$. Stop at the smallest value of $k$ that satisfies (14). In other words we will say that there is dominance at level $k_{d}$ if condition (14) is satisfied at $k=k_{d}$ but not at $k_{d}-1, k_{d}-2, \ldots, 1$. If (14) is not satisfied for any $k<n-1$ then we say that there is no dominance.

Whenever there is dominance at level $k_{d}$, the first $k_{d}$ drops are substantially larger than all of the remaining ones. This means that the mass distribution can be separated in two components: one made of $k_{d}$ massive drops, the other constituted by one or more (usually many) tiny droplets. If on the other hand condition (14) is never satisfied, it is said that there is no dominance and this means

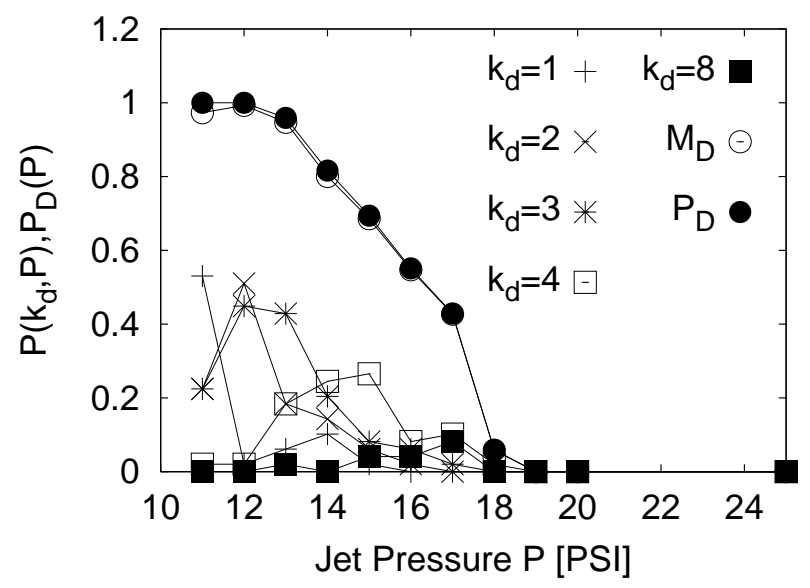

FIG. 10: Probability of dominance $P\left(k_{d}, P\right)$ at $k_{d}=1$ (plusses), 2 (crosses), 3 (asterisks), 4 (empty squares), and 8 (full squares); mass of dominant drops $M_{D}(P)$ (empty circles), and total dominance probability $P_{D}(P)$ (full circles) as a function of pressure $P$.

that the distribution has no distinguishable size scale. In NMF, events where the excited nucleus emits a few small fragments composed of one or two nuclei, remaining otherwise almost unaltered, are called evaporation events. Those events where the original nucleus splits into two large fragments (plus eventually a few much smaller ones) are called fission events, and those for which three large fragments are formed are called ternary fission events. Within the dominance concept we have introduced, the NMF classification mentioned above corresponds to $k_{d}=1,2$ and 3 respectively.

In order to apply these ideas to our experimental data, for each pressure $P$ we determine the probability $P_{D}\left(k_{d}, P\right)$ that there is dominance at level $k_{d}$, by averaging over fragmentation events (typically fifty) at pressure $P$. The resulting dominance probabilities are displayed in Fig. 10 for several values of $k_{d}$. It is apparent from these data that evaporation $\left(k_{d}=1\right)$ events are predominant at low pressure, next fission $\left(k_{d}=2\right)$ events appear as $P$ is increased, followed by a predominance of ternary fission $\left(k_{d}=3\right)$ and so forth. As shown in Fig. 11, the average dominance level $k_{d}$ grows on approach to $P=18$ PSI, beyond which there is no longer any detectable dominance.

The sum over $k$ of $P_{D}(k, P)$ gives probability $D(P)$ that there is dominance at some level, and is also shown in Fig. 10 together with the average mass $M_{D}(P)$ of all dominant droplets. The fact that $M_{D}(P)$ is roughly equivalent to $D(P)$ indicates that, whenever there is dominance, almost the total mass of the system is contained in the $k_{d}$ dominant drops. The total dominance probability $D(P)$ goes to zero at around $18 P S I$, in a way that is consistent with all previously mentioned evidence indicating a critical point around that pressure. Of course the precise numerical values of the dominance observables $P_{D}(k, P)$ depend on the arbitrary choice of the 


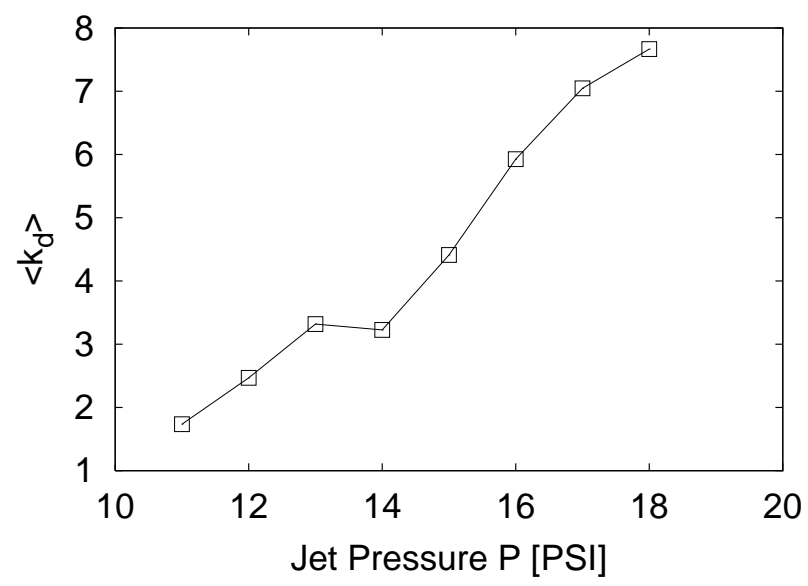

FIG. 11: Average number of dominant drops, versus pressure. For pressures larger than 18PSI, no dominance was ever detected.

dominance parameter $\gamma$, but we have checked that the fact that the dominance probability goes to zero around 18 PSI holds qualitatively unaltered for $\gamma$ ranging from 0.8 to 0.95 . We also explored a different definition of dominance, namely replacing (14) with

$$
x_{k}>\gamma x_{k+1} .
$$

This means that a comparison is established between each mass-sorted droplet and the next, stopping at the first level at which the ratio between them is larger than a given prespecified factor. The use of definition (15) instead of (14) did not alter our findings qualitatively.

We thus feel justified to conclude, based on the data presented in this section, that there is a dominance transition in the neighborhood of $P_{c}=18$ PSI. On the lowpressure side of this transition, a few large (dominant) drops contain most of the original mass, and there is a size-gap separating these large drops from the accompanying "mist" of tiny droplets. Beyond $P_{c}$, on the other hand, one has a collection of droplets which populate homogeneously the whole range of sizes without a systematic size gap.

\section{DISCUSSION}

We have presented a statistical analysis of droplet-size distributions obtained when a liquid drop is broken by a sudden gas jet blow. A flatbed scanner and image processing software were used to automatize the process of counting and measuring the resulting fragments, enabling us to collect statistics for breakdown processes resulting in up to tens of thousands of tiny droplets. The resulting size-distributions $N(x)$, displayed in Fig. 4, can be fitted by a simplified Fisher-model expression (Eq. (4)) and it was found that $N(x)$ behaves like a pure powerlaw only at $P_{c} \approx 17 \mathrm{PSI}$, providing the first hint for the existence of a phase transition there. Further evidence suggesting a phase transition is given by a deep minimum in the apparent Fisher exponent $\tau$ (Fig. 6), and by a sharp slope discontinuity in the relation between pressure and surface increase (Fig. 17), both of them occurring at $P_{c}$. We argued that it is not possible to identify $P_{c}$ as a percolation critical point, as was done in NMF [11, 12] and Fracture [22, 23, 49] experiments, because: a) $\tau^{\text {min }} \approx 1$ (Fig. 6) is far from the value 2.3 that would be expected for a percolative transition, and b) in our case, the mass $Z_{\max }$ of largest fragment does not become zero at $P_{c}$, but instead behaves as $P^{-3 / 2}$ in the whole pressure range considered (See Fig. 8). Low values of the apparent exponent $\tau$ have been previously reported in NMF experiments [1, 2, 67].

Furthermore, the behavior of the largest and average fragments, in our experiments, was found to be consistent with recent predictions of Ashurst and Holian (AH) [3, 4], if the original droplet is assumed to expand at rate $\eta$ that is proportional to $P$ (Fig. 8).

Analysis of moment correlations through a scatter plot (see Fig. 9) reveals a behavior similar to the one already found by Campi [50] in NMF experiments, and which was invoked as evidencing percolative critical behavior. We showed, however, that the shape of the scatter plot displayed in Fig. 9, i.e. the existence of a maximum in the values of $S_{2}(Z)$, is in no way related to criticality, since it is there even for a simple, non-critical, partitioning model, as discussed in Section IV C.

We introduced the concept of "dominance" in an attempt to quantify the observed fact that a few large droplets contain most of the original mass at low pressures, while no dominant droplets are observed at larger pressures. The dominance probability, as we defined it, although is dependent upon an arbitrary parameter $\gamma$, shows a sharp fall (Fig. 10) to zero at a critical pressure $P_{c} \approx 18$ PSI, that is roughly independent of $\gamma$. Of course the precise value of $P_{c}$ is not interesting by itself, since it will depend upon many parameters like the volume and viscosity of the original droplet, the amount of gas contained in the pressurized chamber, the diameter of the outlet (and thus the exhaust velocity), etc, whose variation we did not consider in this experiment. Conceptually more interesting is the fact that, as the energy available to break the droplet increases, a sharp transition occurs between two breakdown regimes, and that this transition can be characterized by statistical methods as discussed in Section IV

Future work could include the use of fast imaging techniques in order to put these results in the context of what is known about rupture modes in the field of sprays [32, 68, 69, 70]. The following picture emerges for isolated droplets subject to a sudden gas blow. At low excitation intensity (low Weber number) the prevalent rupture mechanism is vibrational breakup, whereby collective oscillations break the drop into a small number of roughly equal-sized droplets. Upon increasing the breakdown energy, bag breakup appears, which is characterized by the deformation of the drop into a torus- 
shaped rim, which subsequently disintegrates. A variant of this is called bag-and-stamen breakup, in which a central "stamen" is also formed. Next come shear breakup, where small droplets are continuously stripped off the rim of the drop, and catastrophic breakup, where strong surface waves disintegrate the drop violently. The limit Weber number between vibrational and bag breakup is around 10. At 20-60 shear breakup appears, and at 1000 catastrophic breakup.

Since our experiments were conducted on droplets hanging from a thin glass tip, the presence of which certainly modifies the gas flow and the ensuing breakup modes, we do not expect a direct correspondence with the above classification, although a comparison with the rupture modes of free droplets would certainly be instructive.
Also interesting is to establish a comparison of our results with recent analyses of NMF data in the context of phase transitions 11, 12]. Common characteristics are: a sharp minimum in the apparent $\tau$ exponent 1, 2, 11, a strong curvature change in a log-log plot of $N(x)$ [53, 56], and the fact that $N(x)$ is, to a good approximation, a pure power law at criticality [1, 2, 11, [53, 56].

\section{Acknowledgments}

This work has been partially supported by Conacyt, México, under Grants No. 46709-F and 48783-F.
[1] A. D. Panagiotou, M. W. Curtin, H. Toki, D. K. Scott, and P. J. Siemens, Phys. Rev. Lett. 52, 496 (1984).

[2] M. Mahi, A. T. Bujak, D. D. Carmony, Y. H. Chung, L. J. Gutay, A. S. Hirsch, G. L. Paderewski, N. T. Porile, T. C. Sangster, R. P. Scharenberg, et al., Phys. Rev. Lett. 60, 1936 (1988).

[3] W. T. Ashurst and B. L. Holian, Phys. Rev. E 59, 6742 (1999).

[4] W. T. Ashurst and B. L. Holian, J. Chem. Phys. 111, 2842 (1999).

[5] P. Pourghahramani and E. Forssberg, Miner. Process Extr. Metall. Rev. 26, 167 (2005).

[6] G. Delagrammatikas and S. Tsimas, Chem. Eng. Commun. 191, 1362 (2004).

[7] M. Rieu and G. Sposito, Soil Sci. Soc. Am. J. 55, 1231 (1991).

[8] E. Perfect, Eng. Geol. 48, 185 (1997).

[9] W. W. Emerson, Aust. J. Soil Res. 41, 1089 (2003).

[10] H. Millan, M. Gonzalez-Posada, M. Aguilar, J. Dominguez, and L. Cespedes, Geoderma 117, 117 (2003).

[11] J. Richert and P. Wagner, Phys. Rep.-Rev. Sec. Phys. Lett. 350, 3 (2001).

[12] C. B. Das, S. Das Gupta, W. G. Lynch, A. Z. Mekjian, and M. B. Tsang, Phys. Rep.-Rev. Sec. Phys. Lett. 406, 1 (2005).

[13] W. K. Brown, R. R. Karpp, and D. E. Grady, Astrophys. Space Sci. 94, 401 (1983).

[14] L. Oddershede, A. Meibom, and J. Bohr, Europhys. Lett. 43, 598 (1998).

[15] A. Z. Mekjian and S. J. Lee, Phys. Rev. A 44, 6294 (1991).

[16] A. Z. Mekjian, Phys. Rev. A 44, 8361 (1991).

[17] E. Villermaux, P. Marmottant, and J. Duplat, Phys. Rev. Lett. 92 (2004).

[18] P. H. Marmottant and E. Villermaux, J. Fluid Mech. 498, 73 (2004).

[19] R. Schmehl, H. Rosskamp, M. Willmann, and S. Wittig, Int. J. Heat Fluid Flow 20, 520 (1999).

[20] O. Sotolongocosta, E. Lopezpages, F. Barrerastoledo, and J. Marinantuna, Phys. Rev. E 49, 4027 (1994).

[21] O. Sotolongo-Costa, R. Grau-Crespo, and C. TralleroHerrero, Rev. Mex. Fis. 44, 461 (1998).
[22] F. K. Wittel, F. Kun, H. J. Herrmann, and B. H. Kroplin, Phys. Rev. E $\mathbf{7 1}$ (2005).

[23] F. Wittel, F. Kun, H. J. Herrmann, and B. H. Kroplin, Phys. Rev. Lett. 93 (2004).

[24] J. A. Astrom, Adv. Phys. 55, 247 (2006).

[25] Y. X. Xiao, C. F. Lee, and S. J. Wang, Eng. Geol. 66, 221 (2002).

[26] M. Joshi and J. K. Ra, J. Geol. Soc. India 61, 664 (2003).

[27] R. Delannay, G. LeCaer, and R. Botet, J. Phys. A-Math. Gen. 29, 6693 (1996).

[28] P. Barak, C. A. Seybold, and K. McSweeney, Soil Sci. Soc. Am. J. 60, 72 (1996).

[29] T. Ishii and M. Matsushita, J. Phys. Soc. Jpn. 61, 3474 (1992).

[30] W. H. Chou, L. P. Hsiang, and G. M. Faeth, Int. J. Multiph. Flow 23, 651 (1997).

[31] L. P. Hsiang and G. M. Faeth, Int. J. Multiph. Flow 19, 721 (1993).

[32] L. P. Hsiang and G. M. Faeth, Int. J. Multiph. Flow 18, 635 (1992).

[33] E. Villermaux, New J. Phys. 6 (2004).

[34] W. K. Brown and K. H. Wohletz, J. Appl. Phys. 78, 2758 (1995).

[35] T. M. Zobeck, T. E. Gill, and T. W. Popham, Earth Surf. Process. Landf. 24, 943 (1999).

[36] M. Arias, E. Lopez, and T. Barral, Clay Min. 32, 3 (1997).

[37] L. Oddershede, P. Dimon, and J. Bohr, Phys. Rev. Lett. 71, 3107 (1993).

[38] P. BAK, C. TANG, and K. WIESENFELD, Phys. Rev. A 38, 364 (1988), ISSN 1050-2947.

[39] A. Meibom and I. Balslev, Phys. Rev. Lett. 76, 2492 (1996).

[40] Z. Tavassoli and G. J. Rodgers, Phys. Lett. A 256, 272 (1999).

[41] O. Sotolongo-Costa, A. H. Rodriguez, and G. J. Rodgers, Physica A 286, 638 (2000).

[42] Z. Tavassoli and A. E. Shirvani, Physica A 286, 29 (2000).

[43] P. M. C. de Oliveira, T. J. P. Penna, A. R. Lima, J. Sa Martins, C. F. Moukarzel, and C. A. F. Leite, Trends Stat. Phys. 3, 137 (2000).

[44] P. M. C. de Oliveira, C. A. F. Leite, C. V. Chianca, 
J. S. S. Martins, and C. F. Moukarzel, Physica A 375, 375 (2007).

[45] O. SotolongoCosta, Y. MorenoVega, J. J. LloverasGonzalez, and J. C. Antoranz, Phys. Rev. Lett. 76, 42 (1996).

[46] F. Kun and H. J. Herrmann, Phys. Rev. E 59, 2623 (1999).

[47] J. A. Astrom, B. L. Holian, and J. Timonen, Phys. Rev. Lett. 84, 3061 (2000).

[48] H. Katsuragi, D. Sugino, and H. Honjo, Phys. Rev. E 68 (2003).

[49] H. J. Herrmann, F. K. Wittel, and F. Kun, Physica A 371, 59 (2006).

[50] X. Campi, J. Phys. A-Math. Gen. 19, L917 (1986).

[51] G. Fai, L. P. Csernai, J. Randrup, and H. Stocker, Phys. Lett. B 164, 265 (1985).

[52] J. Pochodzalla, T. Mohlenkamp, T. Rubehn, A. Schuttauf, A. Worner, E. Zude, M. Begemannblaich, T. Blaich, H. Emling, A. Ferrero, et al., Phys. Rev. Lett. 75, 1040 (1995).

[53] M. Belkacem, V. Latora, and A. Bonasera, Phys. Rev. C 52, 271 (1995).

[54] L. G. Moretto, L. Phair, R. Ghetti, K. Tso, N. Colonna, W. Skulski, G. J. Wozniak, D. R. Bowman, N. Carlin, M. Chartier, et al., Phys. Rev. Lett. 76, 372 (1996).

[55] J. C. Pan, S. Das Gupta, and M. Grant, Phys. Rev. Lett. 80, 1182 (1998).

[56] F. Gobet, B. Farizon, M. Farizon, M. J. Gaillard, J. P. Buchet, M. Carre, and T. D. Mark, Phys. Rev. Lett. 8720, art. no. (2001).

[57] J. B. Elliott, L. G. Moretto, L. Phair, G. J. Wozniak, L. Beaulieu, H. Breuer, R. G. Korteling, K. Kwiatkowski, T. Lefort, L. Pienkowski, et al., Phys. Rev. Lett. 88 (2002).

[58] M. D'Agostino, M. Bruno, F. Gulminelli, R. Bougault, F. Cannata, P. Chomaz, F. Gramegna, N. Le Neindre, A. Moroni, and G. Vannini, Nucl. Phys. A 734, 512
(2004).

[59] M. E. Fisher, Physics-New York 3, 255 (1967).

[60] D. Stauffer and A. Aharony, Introduction to Percolation Theory (Taylor and Francis, Bristol, 1994), 2nd ed.

[61] Y. G. Ma, J. B. Natowitz, R. Wada, K. Hagel, J. Wang, T. Keutgen, Z. Majka, M. Murray, L. Qin, P. Smith, et al., Phys. Rev. C $\mathbf{7 1}$ (2005).

[62] M. D'Agostino, M. Bruno, F. Gulminelli, R. Bougault, F. Cannata, P. Chomaz, F. Gramegna, I. Iori, N. Le Neindre, G. V. Margagliotti, et al., Nucl. Phys. A 724, 455 (2003).

[63] P. T. Reuter and K. A. Bugaev, Phys. Lett. B 517, 233 (2001).

[64] J. B. Elliott, L. G. Moretto, L. Phair, G. J. Wozniak, S. Albergo, F. Bieser, F. P. Brady, Z. Caccia, D. A. Cebra, A. D. Chacon, et al., Phys. Rev. Lett. 85, 1194 (2000).

[65] J. Schmelzer, G. Ropke, and F. P. Ludwig, Phys. Rev. C 55, 1917 (1997).

[66] P. Finocchiaro, M. Belkacem, T. Kubo, V. Latora, and A. Bonasera, Nucl. Phys. A 600, 236 (1996).

[67] A. D. Panagiotou, M. W. Curtin, and D. K. Scott, Phys. Rev. C 31, 55 (1985).

[68] M. Pilch and C. A. Erdman, Int. J. Multiph. Flow 13, 741 (1987).

[69] D. D. Joseph, J. Belanger, and G. S. Beavers, Int. J. Multiph. Flow 25, 1263 (1999).

[70] C. S. Lee and R. D. Reitz, Atom. Sprays 11, 1 (2001).

[71] This is about the maximum volume that can be held by the liquid's surface tension.

[72] Using Adobe Photoshop

[73] http://rsb.info.nih.gov/ij/

[74] The average considered here to is not the number average $1 / N_{\text {drops }}$ but the mass average $\sum_{i} x_{i}^{2} / \sum_{i} x_{i}=M_{2}$. 